\title{
Regenerative abilities of mesenchymal stem cells through mitochondrial transfer
}

Swati Paliwal ${ }^{1}$, Rituparna Chaudhuri ${ }^{1}$, Anurag Agrawa ${ }^{2^{*}}$ and Sujata Mohanty ${ }^{1^{*}}$

\begin{abstract}
The past decade has witnessed an upsurge in studies demonstrating mitochondrial transfer as one of the emerging mechanisms through which mesenchymal stem cells (MSCs) can regenerate and repair damaged cells or tissues. It has been found to play a critical role in healing several diseases related to brain injury, cardiac myopathies, muscle sepsis, lung disorders and acute respiratory disorders. Several studies have shown that various mechanisms are involved in mitochondrial transfer that includes tunnel tube formation, micro vesicle formation, gap junctions, cell fusion and others modes of transfer. Few studies have investigated the mechanisms that contribute to mitochondrial transfer, primarily comprising of signaling pathways involved in tunnel tube formation that facilitates tunnel tube formation for movement of mitochondria from one cell to another. Various stress signals such as release of damaged mitochondria, mtDNA and mitochondrial products along with elevated reactive oxygen species levels trigger the transfer of mitochondria from MSCs to recipient cells. However, extensive cell signaling pathways that lead to mitochondrial transfer from healthy cells are still under investigation and the changes that contribute to restoration of mitochondrial bioenergetics in recipient cells remain largely elusive. In this review, we have discussed the phenomenon of mitochondrial transfer from MSCs to neighboring stressed cells, and how this aids in cellular repair and regeneration of different organs such as lung, heart, eye, brain and kidney. The potential scope of mitochondrial transfer in providing novel therapeutic strategies for treatment of various pathophysiological conditions has also been discussed.
\end{abstract}

Keywords: Mesenchymal stem cells, Mitochondrial transfer mechanism, Regenerative medicine

\section{Background}

Recent advancements in regenerative medicine have capitalized on using stem cells for cellular repair and regeneration. Mesenchymal stem cells (MSCs) have emerged as promising candidates to treat various diseases owing to their unique characteristic properties such as self-renewal, immuno-suppressive potential and ability to trans-differentiate. They are easily available from various tissue sources such as bone marrow, adipose tissue, dental, umbilical cord etc. with low risk of rejection and several clinical trials have provided evidences of their beneficial roles. Interestingly, apart from through engraftment, MSCs are also being shown to exert their actions through paracrine mechanisms, thus repairing cellular damage [1-3]. These paracrine effects

\footnotetext{
* Correspondence: a.agrawal@igib.in; drmohantysujata@gmail.com

${ }^{2}$ Molecular Immunogenetics Laboratory and Centre of Excellence for

Translational Research in Asthma \& Lung disease, CSIR-Institute of Genomics and Integrative Biology, Delhi 110007, India

'Stem Cell Facility, DBT Centre of Excellence for Stem Cell Research, All India Institute of Medical Sciences, New Delhi 110029, India
}

through which MSCs mediate repair can be through release of several immuno-modulatory factors, microvesicles, microRNAs, exosomes and mitochondrial transfer [4]. Mitochondrial dysfunction is associated with a majority of degenerative diseases like ischemic heart diseases, lung disorders, stroke, brain injury and several degenerative diseases including cardiomyopathy, Parkinson's and Alzheimer's [5-7]. Thus, mitochondrial transfer alone has appeared as a great therapeutic strategy as it can restore the bioenergetic needs of damaged cells. The transfer of healthy mitochondria appears to be an effective reparative strategy to rejuvenate several kinds of damaged cells such as epithelial cells, endothelial cells, cardiomyocytes and cells of other origins as well. In this review article, we will be discussing the role of mitochondrial transfer through MSCs and how it aids in repair and regeneration of injured and damaged cells under stress. 


\section{Discovery of mitochondrial transfer and its impact on regenerative medicine}

The transfer of mitochondria, which is a DNA containing organelle, is quite an intriguing phenomenon. Initial discovery of this process was observed in a co-culture system when mitochondrial DNA from donor human MSCs was found in recipient cells devoid of mitochondria [8]. Human epithelial cells when treated with ethidium bromide for long duration lost their mitochondrial activity. These human epithelial cells devoid of mitochondria (A549 rho cells) were co-cultured with healthy MSCs. It was observed that mitochondria respiration of epithelial cells could be restored when co-cultured with MSCs through mitochondrial transfer. This was demonstrated by presence of DsRed2 tagged mitochondria in MSCs and observed by time-lapse photo microscopy. It was also confirmed that uptake of mitochondria was facilitated by active and not passive transfer [8]. Dysfunctional mitochondria can lead to cellular damage and apoptosis, thus a rescue mechanism wherein healthy mitochondria can be transferred has shown tremendous potential. Other cells such as fibroblast and somatic cells have also shown the ability to transfer mitochondria. Mitochondrial donation by MSCs is a faster and more economical physiological process in the cell to replace dysfunctional mitochondria in diseased cells/ tissue, as compared to the mitochondrial biogenesis and thus, appears as an efficient means to attenuate a disease conditions. During the time that Spees et al. published their work, many research groups had simultaneously acknowledged and identified the formation of tunnel tube formation for cell-cell communications [9-12]. Rustom et al., had demonstrated de novo formation of multiple tunnel tubes leading to complex networks between cells and facilitating transfer of membrane vesicles and organelles between cells [9]. These tunnel tubes comprise of F-actin based connections between distant cells and exist in diverse morphologies carrying multiple cargos and signals between cells [13]. These studies were followed by a plethora of studies that provided evidence for mitochondrial transfer between MSCs and damaged cells of varied origins [1418]. The discovery of remarkable mitochondrial transfer ability of MSCs to cells with dysfunctional mitochondria paved way for numerous studies [14]. MSCs from different tissue sources like bone marrow, adipose, and Wharton's jelly have now been shown to transfer mitochondria to various damaged cells, like osteosarcoma cells, that aid in the restoration of their respiratory activities [19]. Table 1 provides a list of studies performed using different sources of stem cells in different conditions, described in detail in later sections of the review.

\section{Mitochondrial bioenergetics status in MSCs}

Mitochondria play important roles in oxidative phosphorylation, ATP generation, and cellular apoptosis. At the mitochondrial inner membrane, electron transport chain is involved in the generation of energy, where oxygen acts as the electron acceptor. During oxidative phosphorylation, electron leak from mitochondrial complexes in electron transport chain results in partial reduction of oxygen and generation of reactive oxygen species (ROS). Antioxidant enzymes present in the cells metabolize toxic intermediates to maintain cellular homeostasis. However, these control mechanisms are not effective in dysfunctional mitochondria and accumulation of ROS results in oxidative damage in cell. In a bio-energetically active cells, increased ROS production triggers dynamic changes in mitochondria and mitochondrial fragmentation occurs through fission and autophagy. Under stress, these cellular mechanisms are unable to maintain mitochondrial homeostasis resulting in dysfunctional mitochondria. In contrast, MSCs maintain themselves in the glycolytic state, and switch from glycolysis to oxidative phosphorylation upon differentiation to meet energy needs of the cells. Mitochondria in MSC exist in dormant state and display low activity status due to lesser energy demands. However upon differentiation, an increase in mtDNA copy number, high levels of respiratory enzymes and mRNA levels, enhanced oxygen consumption rate and increased levels of intracellular ATP are observed [20, 21]. Extensive restructuring and remodeling of the mitochondria occurs during differentiation or transfer to cells with higher bioenergetics demands. It is well established that mitochondrial biogenesis and aerobic respiration gets unregulated in MSCs during differentiation and oxidative stress. Mitochondrial biogenesis is controlled by proliferator activated receptor gamma coactivator-1 $\alpha$ (PGC-1 $\alpha)$. This further activates nuclear respiratory factors (NRF 1 and NRF 2) and mitochondrial transcription factors (mTFA) which coordinates DNA polymerase $\gamma$ and facilitates replication of mtDNA [22]. This is followed by concomitant increase in intracellular ATP levels and up regulation of oxidative metabolism in MSCs. A complex regulatory network of proteins maintains cellular homeostasis between mitochondrial biogenesis and cellular metabolism of MSCs. This process is further tightly regulated by fission, fusion and mitophagy that impact its shape, size and location in the cell [23-25]. Studies provide evidence that mitochondrial metabolism, cell differentiation, stemness of stem cells and cellular apoptosis are interdependent cellular processes [24, 26-29]. On the basis of energy needs ranging from low to high, mitochondrial shape varies from small, fragmented, circular, undeveloped cristae to larger networks of elongated mitochondria with well-developed cristae, respectively. This transformation eventually impacts mitochondrial number, morphology, cellular metabolism and mitochondrial activity $[6,20,30,31]$. Stem cells maintain low levels of cellular ATP, low levels of oxidative damage levels and tightly regulated redox balance as opposed to differentiated 
Table 1 Mitochondrial transfer from Different Tissue Specific MSCs to Recipient Cells of Different Origins

\begin{tabular}{|c|c|c|c|c|c|}
\hline S. No & MSCs Type & Recipient Cell & Mode of Transfer & Action & References \\
\hline 1 & BM-MSCs & A549 Cell Line & $\begin{array}{l}\text { Cellular Contact and } \\
\text { Cytoplasmic Projections }\end{array}$ & Restored Aerobic Respiration & [8] \\
\hline 2 & BM-MSCs & $\begin{array}{l}\text { Pulmonary Alveoli in mouse } \\
\text { lungs }\end{array}$ & $\begin{array}{l}\text { Connexin } 43 \text { alveolar } \\
\text { attachment and } \\
\text { microvesicles }\end{array}$ & Protection against acute lung injury & [15] \\
\hline 3 & $\begin{array}{l}\text { BM-MSCs } \\
\text { and iPSCs }\end{array}$ & Airway epithelial cells & Tunnel Tubes & Rescue cigarettesmoke induced damage & [20] \\
\hline 4 & BM-MSCs & Bronchial Epithelial Cells & Tunnel Tubes and Miro1 & Rescue Bronchial Epithelial Injury & [21] \\
\hline 5 & BM-MSCs & H9c2 cardiomyocyte cell line & Tunnel Tubes & Rescue cardiomyocytes in ischemia model & [22] \\
\hline 6 & BM-MSCs & Cardiomyocytes & Tunnel Tubes & $\begin{array}{l}\text { Repair damaged cardiomyocytes in failed } \\
\text { myocardium }\end{array}$ & [23] \\
\hline 7 & BM-MSCs & Cardiomyoblasts & $\begin{array}{l}\text { Cell-Cell Connection and } \\
\text { Tubular Connections }\end{array}$ & Rescue Cells from Ischemic Damage & [24] \\
\hline 8 & BM-MSCs & Kidney Tubular Epithelial cells & Microvesicles & Recovery from acute kidney injury & [25] \\
\hline 9 & BM-MSCs & Rat Cortical Neuronal Cells & Tunnel Tubes and Miro1 & Neuroprotective & [26] \\
\hline 10 & BM-MSCs & Macrophages & Tunnel Tubes & Anti-Bacterial Activity & [27] \\
\hline 11 & BM-MSCs & Macrophages & Extracellular Vesicles & $\begin{array}{l}\text { Regulation of mitochondrial dynamics } \\
\text { and Mitophagy }\end{array}$ & [28] \\
\hline 12 & BM-MSCs & Acute myeloid leukemic cells & Cell-Cell Contact & Survival of Myeloid Cells & [18] \\
\hline 13 & BM-MSCs & $\begin{array}{l}\text { MDA-MB231 breast carcinoma } \\
\text { cell line }\end{array}$ & Isolated mitochondria & Enhanced OXPHOS activity & [29] \\
\hline 14 & iPSCs-MSCs & Corneal Epithelial Cells & Tunnel Tubes & $\begin{array}{l}\text { Protection of corneal epithelial cells } \\
\text { against alkali burn }\end{array}$ & [16] \\
\hline 15 & AD- MSCs & Cardiomyocytes & Cell Fusion & $\begin{array}{l}\text { Reprogram adult cardiac cells towards } \\
\text { progenitor like state }\end{array}$ & [30] \\
\hline 16 & WJ-MSCS & $\begin{array}{l}\text { Cells devoid of mitochondria } \\
\text { Osteosarcoma cells }\end{array}$ & Tunnel Tubes & Mitochondria function was rescued & [19] \\
\hline
\end{tabular}

cells [20, 26, 32]. In Fig. 1, we have shown mitochondrial status in transmission electron images of human BMMSCs and human choriocarcinoma cell line JEG-3, to demonstrate a striking difference in the mitochondria of two cells with low and high-energy demands respectively (unpublished data). The mitochondria in MSCs are spherical in shape with underdeveloped cristae, cytoplasmic localization and less in number, owing to lower energy demand (Fig. 1a). In contrast, cell lines, here JEG-3, have higher energy and thus, branched, elongated mitochondria with well-organized and localized in peri-nuclear region (Fig. 1b). These naive mitochondria are transferred from MSCs to cells with dysfunctional mitochondria to with high oxidative energy demands thereby modulating their cellular metabolism. Mitochondria transfer appears to be a lucrative strategy for regenerative processes, as mitochondrial biosynthesis usually take longer than can be afforded by damaged cells in crisis. Thus, mitochondrial transfer is an efficient method for regeneration of injured cells. Although complete mechanisms of mitochondria transfer have not been elucidated yet, but certain studies suggests that mitochondria transfer from MSCs modulate cellular metabolism of recipient cells. Up regulation of mitochondrial respiration and ATP levels along with reduction in oxidative damage has been observed in several studies wherein MSCs have been cocultured with injured cells or tissues [16,33-35]. It is plausible that mitochondria transferred from MSCs modulate cellular bioenergetics by regulating mtDNA replication, maintaining its copy number and regulating other mitochondrial dynamics and cellular processing required for maintenance of mitochondrial homeostasis in cell.

\section{Signals that trigger mitochondrial transfer from mesenchymal stem cells}

The signals that trigger transfer of mitochondria from MSCs have intrigued scientists for a long time. Researchers have postulated theories suggesting that oxidative stress of dysfunctional mitochondria of recipient cell sends environment cues to the MSCs [15, 34]. The local microenvironment of an injured cell has been shown to release physiological cues that trigger transfer of mitochondria. It is compelling to note that bidirectional mitochondrial transfer occurs between MSCs and their microenvironment. Few studies have illustrated that stress signals released by recipient cells include damaged mitochondria, released mtDNA and mitochondrial products that are characterized as damage associated molecular patterns 


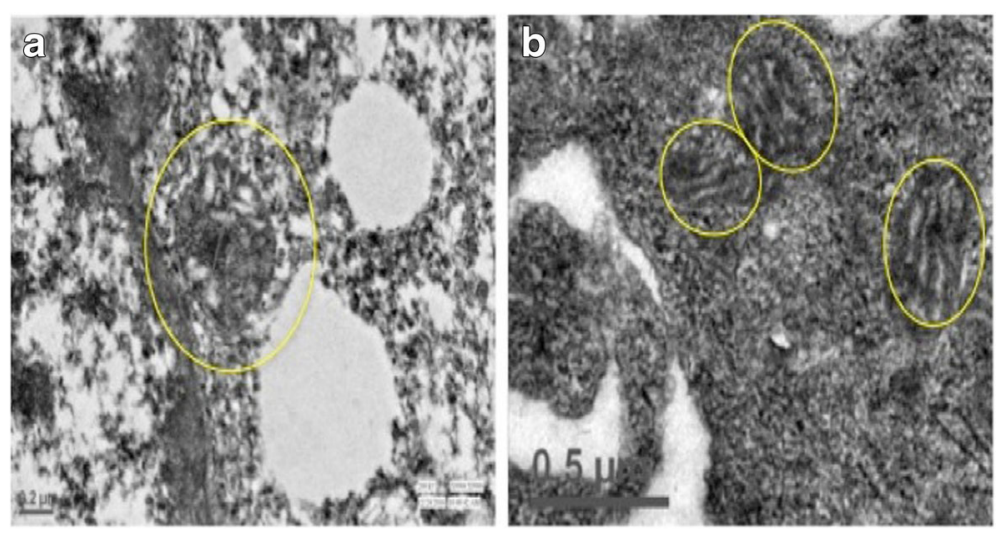

Fig. 1 Striking difference between mitochondria of Human MSCs and JEG-3 Cell Line as observed under Transmission Electron Microscope (TECNAI 200 Kv, Fei, Electron Optics). a Human BM-MSCs cells: Mitochondria are fewer in number, spherical, condensed with underdeveloped cristae and display cytoplasmic localization. Scale Bar: 0.2 um (b) JEG-3 Cell Line, mitochondria are branched, tubular, elongated and well-organized cristae, more in number and peri-nuclear in localization. Scale Bar: $0.5 \mu \mathrm{m}$

(DAMPS) [36-39]. Interestingly, mtDNA released by injured cells are engulfed by MSCs that subsequently triggers the cytoprotective function of MSCs and enhanced mitochondrial biogenesis through retrograde signaling, thereby preparing MSCs for mitochondrial donation [40]. In addition, reactive oxygen species released by cells under oxidative stress and inflammation status have also been postulated to trigger donation of mitochondria [15, 34, 41, 42]. Elevated levels of reactive oxygen species have been found in cells with high energy demands such as in cancer cells, and these usually have been seen to receive mitochondria from surrounding cells to meet its energy demands $[43,44]$. In acute myeloid leukemic cells, NADPH oxidase-2 derived superoxide has been reported to stimulate mitochondrial transfer from BM-MSCs through tunnel tube formation [45]. As discussed previously, that co-culturing MSCs with cells under oxidative stress reversed the oxidative stress state by decreasing mitochondrial ROS and activities of electron transport chain complexes [34]. Thus, it is possible that under retrograde signaling is triggered by $\mathrm{ROS}, \mathrm{Ca}^{2+}, \mathrm{AMP} / \mathrm{ATP}$ and $\mathrm{NAD}^{+} / \mathrm{NADH}$ ratio in cells under oxidative stress. This further stimulates transfer of mitochondria from MSCs to recipient cells. In another study it was shown that calcium dependent mechanism of CD38/cyclic ADP ribose signaling mediated transfer of mitochondria from neurons to astrocytes for recycling and disposal of damaged mitochondria. This was seen to contribute to neuroprotective and neuro-recovery mechanism after stroke [46]. As CD38, catalyzes the synthesis of calcium messenger cyclic ADPribose in mitochondria membranes, its up regulation further increased mitochondria donation in a calcium dependent manner [47]. Another interesting study has shown that loss of cytochrome $\mathrm{c}$ in stressed cells, characterized by caspase 3 activation also triggered mitochondrial donation to rescue
UV-damaged PC12 cells by MSCs in the early stages of apoptosis [48].

In addition, under stress, MSCs are programmed to secrete depolarized mitochondria for their own protection through transcellular mitophagy [49]. In an in vivo model of myocardial infarction, mitochondria released from damaged cells activated anti-apoptotic signals in MSCs. Foreign mitochondria from other cells were shown to be engulfed and degraded in MSCs, leading to induction of cytoprotective enzyme HO-1, and stimulation of mitochondrial biogenesis. This triggered enhanced mitochondrial donation from MSCs to these cells [40]. Phinney et al., have suggested that mitochondrial donation by MSCs is a protective mechanism adopted by MSCs rather than being an altruistic strategy [49]. Simultaneously, mitochondrial DNA that evades autophagy stimulates inflammatory responses mediated by Toll-like Receptor (TLR) 9 and other inflammatory responses, and eventually leads to cellular apoptosis [49-51]. Mitochondria proteins like TFAM and DAMPs including $\mathrm{N}$-formyl peptides, mtDNA, cardiolipin or extracellular ATP are also known to participate in inflammatory responses and trigger transfer of mitochondria from donor to acceptor cells [52]. These studies have shown that various signaling molecules participate in mitochondria transfer from MSCs, but the exact mechanisms and choice of release of a particular signal molecule in a given situation followed by other downstream signals has not been well elucidated.

\section{Mechanisms of mitochondrial transfer}

Mitochondrial transfer has been found to be mediated by different modes that include tunnel tube formation, gap junctions, micro vesicles, cell fusion and transfer of isolated mitochondria [15, 34, 42, 53-56]. Figure 2 diagrammatically illustrates several modes of mitochondrial 


\section{Different Modes of Mitochondria Transfer}

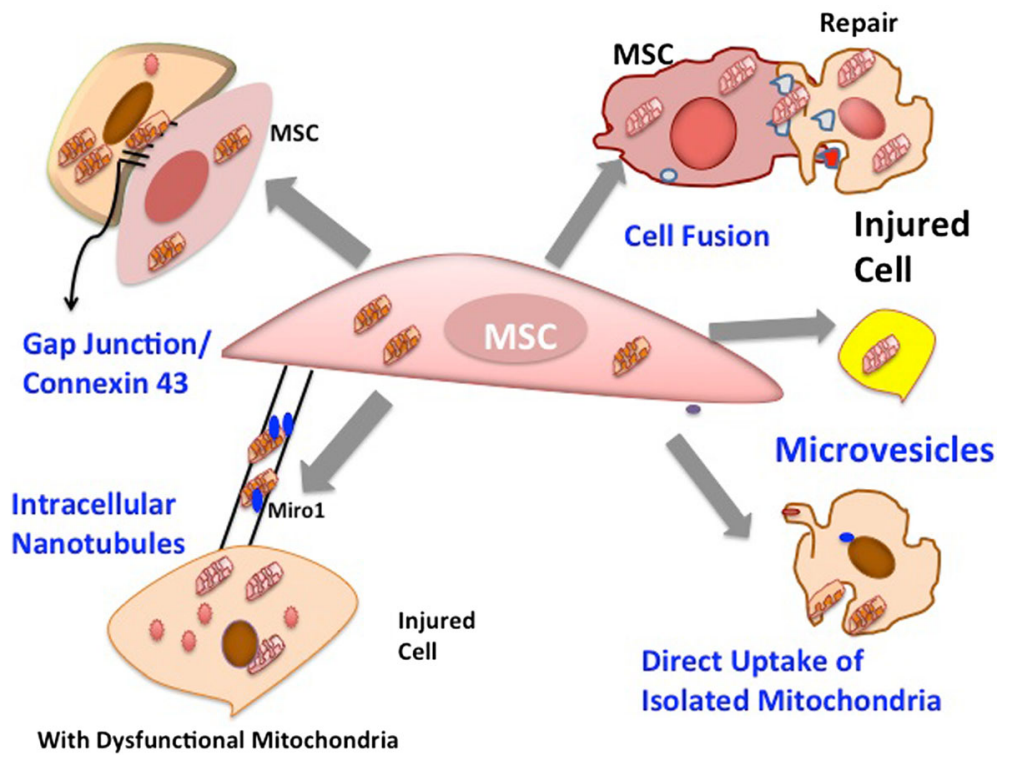

Fig. 2 Different modes of mitochondrial transfer from MSCs to injured or damaged cells. These include transfer through intracellular nanotubes, gap junctions, cell fusion, microvesicles and direct uptake of isolated mitochondria

transfer from MSCs to recipient cells with damaged or dysfunctional mitochondria and Fig. 3 shows overview of mechanism involved in mitochondria trasnfer. A majority of studies illustrate mitochondrial transfer through formation of nanotubes between MSCs and damaged cells. Motor-adaptor protein complexes regulate mitochondrial transport and homeostasis, Miro 1 and Miro 2 are two dynamin related Rho-GTPases that interact with other accessory proteins to allow movement of mitochondria on cytoplasmic tunnel tube extensions that connect two cells. These calcium sensitive proteins bind mitochondria to KLF 5 kinesin motor protein with the help of other accessory proteins like TRAK 1 and TRAK 2, Myo 10 and Myo 19. Together, they form a motoradaptor complex that contributes to mitochondria transport machinery and regulates movement of mitochondria on microtubules. The role of Miro1 was shown in nanotube transport in intercellular mitochondrial transport in a co-culture system of BM-MSCs and alveolar epithelial cells. MSCs overexpressing Miro1 were shown to perform enhanced rescue potential, leading to greater epithelial cell repair [34]. Down regulation of Miro1 expression and inhibition of tunnel tube formation prevented mitochondrial transfer from MSCs to epithelial cells. This study provided supporting evidence that established the significant role of Miro1 in mitochondrial transfer through tunnel tubes [34].

In an interesting study by Zhang et al., iPSCs show superior efficiency than BM-MSCs in mitochondrial transfer in rescue of Anthracycline induced cardiomyopathy due to higher expression of Miro 1 [41]. This was confirmed by various experiments showing that knockdown of Miro1 with short hairpin RNA (shRNA) that led to remarkable decrease in Miro1 expression and subsequent reduction in mitochondrial donation. Nonetheless, overexpression of Miro1 enhanced intracellular mitochondria transfer; thereby providing supporting evidence to confirm that mitochondria transfer is modulated by Miro1 expression. They also investigated tunnel tube formation between iPSCs and cardiomyocytes during mitochondrial transfer. It was shown that no mitochondria transfer occurred when cells were treated with cytochalasin $\mathrm{B}$, that inhibits actin polymerisation and tunnel tube formation. These iPSCs were also found to be more responsive to tumor necrosis factor alpha (TNF $\alpha$ ) that induced tunneling nanotube (TNT) formation in cardiomyocytes by elevating TNF $\alpha$ IP2 expression, which was further, regulated by TNF$\alpha / \mathrm{NF}-\mathrm{kappaB} / \mathrm{TNF} \alpha \mathrm{p} 2$ signaling pathway. A comparative analysis between iPSCs and BM-MSCs showed that iPSCs displayed higher expression of TNFaip2, and more number tunnel tube formation per cell followed by increased mitochondrial transfer. Thus, this study shows that higher expression of both Miro1 and TNFoip2 leads to more mitochondrial transfer and tunnel tube formation and imparts a cell with higher efficiency to transfer mitochondria as in case of iPSCs as compared to BM-MSCs [41].

Similar results have been observed in another study where MSCs transferred mitochondria to corneal epithelial cells through tunnel tube formation via NF-kB/TNFoip2 signaling. This was confirmed by western blot analysis of 


\section{Mechanisms of Mitochondria Transfer}

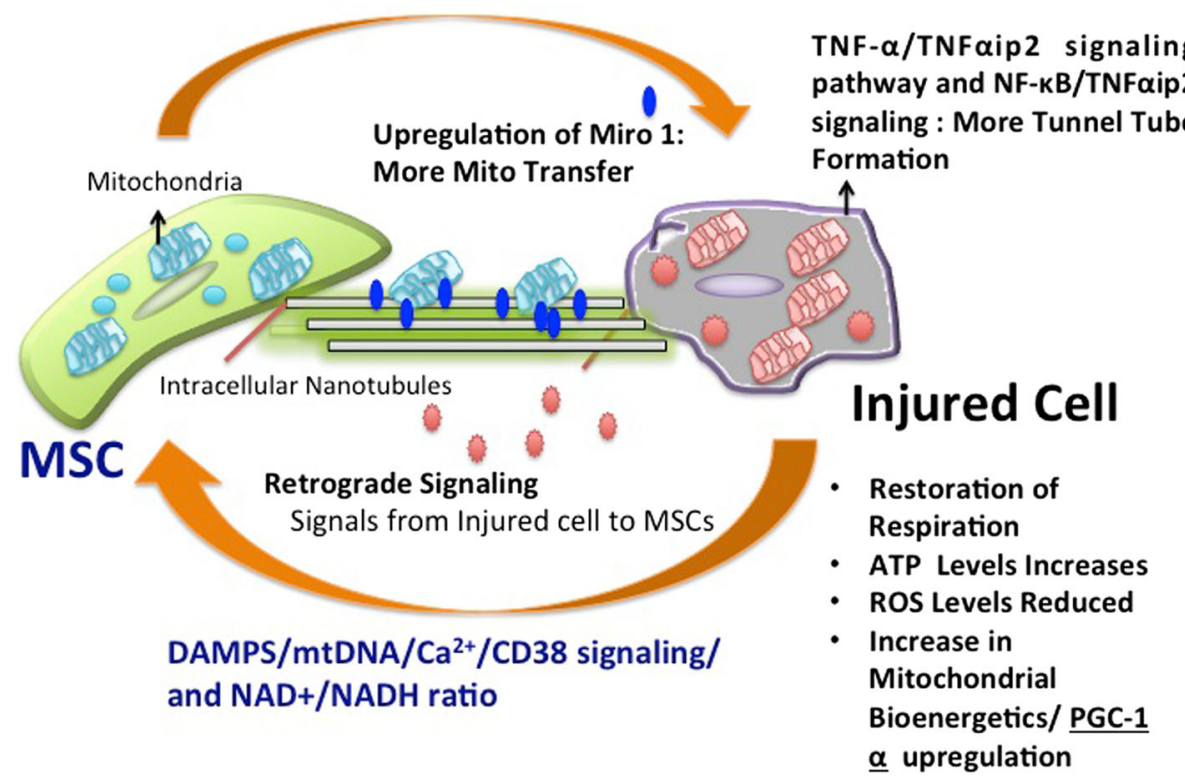

Fig. 3 Mechanisms of Mitochondrial Transfer. An overview of mitochondrial transfer mechanisms has been shown with expression of Miro1 protein and tunnel tube formation signaling pathways along with signaling that trigger mitochondrial release from MSCs to injured cell under stress

protein showing that enhanced levels of phosphorylated of NF- $\kappa B$ subunit $\mathrm{p}-\mathrm{I} \kappa \mathrm{B}$ along with elevated levels of TNF $\alpha$ and TNFoip2 was markedly increased during tunnel tube formation. It was observed that NF-kB inhibitor SC-514 significantly attenuated tunnel tube formation thus; demonstrating the role of NF- $\mathrm{B}$ mediated signaling mechanism in tunnel tube formation. Furthermore, the role of ROS signal in stimulating of tunnel tube formation was validated by using antioxidant scavenger, $\mathrm{N}$-acetylcysteine (NAC) treatment that reduced ROS levels and concomitantly resulted in inhibition of tunnel tube formation and mitochondria transfer between MSCs and CECs [16].

Majority of the studies have shown that mitochondrial transfer is mainly mediated through tunnel tube formation and micro vesicles rather than gap junctions. This could be because most of the studies are carried out in in vitro co-culture system wherein tunnel tube formation is easily observed. However, connexin 43 containing gap junctions channels have been particularly well observed in in vivo acute lung injury mouse model [15]. Sinclair and colleagues have characterized and compared different modes of mitochondrial transfer from different types of MSCs to bronchial epithelial cells [57]. Complete abrogation of mitochondrial transfer was observed by blocking tunnel tube formation by cytochalasin B and inhibition of microvesicles by dynasore [57]. Cytoplasmic transfer was found unaffected by Gap26 inhibitor of connexin 43. However, a cocktail of inhibitors exhibited conspicuous attenuation of mitochondrial transfer and at greater extents suggesting synergy between these modes of mitochondrial transfer [57]. Uptake of mitochondria isolated from stem cells has also been observed in case of MDA-MB231 cell lines and cardiomyocytes [54, 58]. Although, the exact mechanism of mitochondrial uptake is yet unknown but few studies have suggested that internalization of mitochondria is mediated by actin-dependent cellular mechanisms [53, 58, 59]. Different modes of transfer have been observed under various pathophysiological conditions as illustrated further in this review. However, the basis for choice of mode of mitochondrial transfer under a particular condition is unclear. Though, it is quite possible that the proximity of damaged cells to MSCs and the environmental condition might act as contributing factors regulating the mode of transfer for mitochondrial transfer.

In a study by Spees et al., BM-MSCs were shown to transfer functional mitochondria to rho $\phi$ cells devoid of functional mitochondria. Careful examination of these rescued cells excluded cell fusion as one of the main mechanisms of mitochondrial transfer. It was also shown that active formation of TNTs or vesicular transfer of mitochondria, but not passive uptake of mitochondrial fragments, resulted in functional complementation. Islam et al showed that formation of connexin-43 gap junction channels is essential for mitochondrial transfer from BMMSCs to alveolar epithelial cells, thus increasing epithelial ATP production. BM-MSCs expressing dysfunctional connexin 43 could not adhere to alveolar epithelium, and also 
could not transfer mitochondria. Cytosolic calcium ions and cellular bioenergetics levels of ATP and glucose are also considered as major players in regulating movement of mitochondria [42]. Further comprehensive studies and elucidations of mitochondrial transfer mechanisms are required to better understand the signaling pathways and molecules involved in this process to employ mitochondrial transfer to many therapeutic applications.

\section{Mitochondrial transfer in regeneration of diseased tissues} Mitochondria transfer from MSCs has been studied in various disease models that have not only provided evidence for its significance in regenerative medicine but also helped to gain insight on mitochondria transfer mechanisms. Different preferred mechanism and modes have been observed on the basis of concerned recipient cells and stress condition. In lung cells, renal cells, corneal epithelial cells and brain cortical cells, mitochondria transfer has occurred mostly through tunnel tube formation. However, cell fusion and reprogramming of progenitor cells have been observed in cardiac cells. Micro vesicle formation has been observed predominantly in studies where immunemechanisms are involved as in the case of anti-microbial activity. Nonetheless, remarkable restoration of cellular bioenergetics and reduction in oxidative stress was observed in all the studies that demonstrates that mitochondria transfer from MSCs plays critical role in cellular repair and regeneration. Also, another therapeutic strategy that targets inhibition of tunnel tube formation and mitochondrial transfer in case of tumors has also been discussed.

\section{Lung diseases}

Interestingly, mitochondrial transfer has been studied mostly in lung diseases. One of the remarkable works includes study carried out by Islam et al., wherein they found that BM-MSCs transfer mitochondria to pulmonary alveoli contributing to protection from acute lung injury [15]. This study reported mitochondrial transfer in intact lungs in in vivo mice model treated with lipopolysaccharide (LPS). It was noted that human or mice BMMSCs instilled in mice airways were able to transfer mitochondria and repair mitochondrial bioenergetics in lungs. The mitochondrial transfer occurred over a period of $24 \mathrm{~h}$ in the lung epithelium and was supported by the expression of connexin 43 that mediated mitochondrial transfer through nanotubes and microvesicles in a calcium dependent manner. Mitochondrial transfer led to increased ATP concentrations in the recipient cells suggesting its role in bioenergetics. This study was followed by another remarkable discovery of role of Miro1, a mitochondrial Rho-GTPase that regulated mitochondrial transfer from MSCs to lung epithelial cells through connecting nanotubes [34]. In this study, Ahmad et al., reported that over expression of Miro1 enhanced mitochondrial transfer whereas its knockdown lead to the loss of rescue efficacy of MSCs. This study was performed in rotenone induced lung injury mouse model and further confirmed in allergen induced asthma models. Another study investigated the effect of mitochondrial transfer in rat models exposed to cigarette smoke for 56 days that induced lung damage and manifestation of chronic obstructive pulmonary disease [60]. It was reported that BM-MSCs mitigated the damage by transferring mitochondria to lung epithelium. Similar to results of previous studies, they also found significant mitochondrial transfer occurred after $24 \mathrm{~h}$ of MSC and BEAS-2B coculture followed by subsequent elevation in ATP levels through tunnel tube formation. However, further research along these lines is warranted as the reasons for difference are vastly unknown and a comparative analysis of mitochondrial transfer abilities of stem cells from different sources remain unexplored. Li et al., have also suggested that mitochondrial dysfunction is observed in case of prolonged inflammation and stem cells can transfer mitochondria to mitigate inflammation indicative of their rescue potential by promoting anti-inflammatory effects.

\section{Cardiac tissues}

Mitochondrial transfer between endothelial progenitor cells were reported to regenerate and repair damaged myocardium [53, 61]. Vallabhaneni et al, reported the role of functional mitochondria transfer and intercellular communication between MSCs and vascular smooth muscle cells through intercellular tunnel tube bridges and not necessarily through differentiation, as claimed by earlier studies [62]. This study was also validated in another investigation that demonstrated cell-to-cell connection between endothelial cells and cardiomyocytes leading to mitochondrial transfer through nanotube formation, independent of cellular or nuclear fusion $[35,53,63]$. Cellular processes involved in mitochondrial dynamics play critical role in cardiovascular systems [64]. Dysfunctional mitochondria are predominant in cardiac disorders such as ischemia, myocardial infarction and other cardiomyopathies are found to be ameliorated by administration of healthy mitochondria [65-68]. Acquistapace et al., cocultured human adipose stem cells with mouse cardiomyocytes and confirmed formation of intercellular F-actin connections showing mitochondrial transfer and partial cell fusion as mechanism of cell repair [33]. Cross-talk between stem cells and cardiomyocytes through tunnel tubes was further confirmed by other studies suggesting mitochondrial transfer as futuristic therapeutic strategy $[17,67,69]$. Figure 4 shows transfer of mitochondria stained with MitoTracker Green from human BM-MSCs to U87-MG cell line and human cardiomyocytes through tunnel tubes stained with phalloidin red. The cardiomyocytes were treated with antimycin $\mathrm{A}(100 \mu \mathrm{M})$ to introduce oxidative stress condition. Laser-patterned chips 

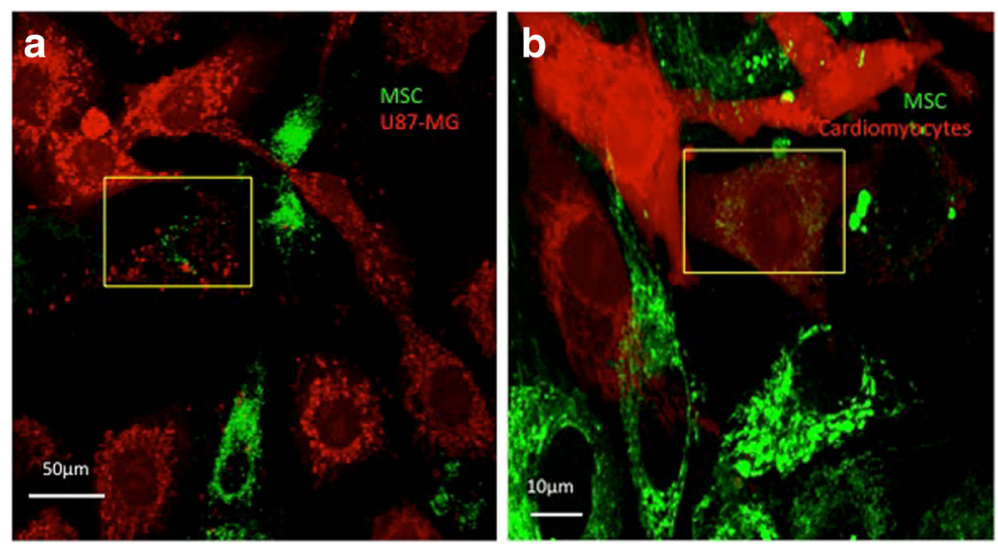

Fig. 4 Mitochondria stained with MitoTracker Green FM (Thermo Fisher Scientific) in human BM-MSCs were transfered to Antimycin A treated (a) U87-MG and (b) rat cardiomyocyte. Confocal imaging was done on Leica TCS SP5 using software LAS AF

have also been devised that further shed light on mitochondrial transfer and cellular contact between MSCs and cardiomyocytes [70]. These studies have shown that mitochondrial transfer from stem cells can prove to be an effective therapeutic strategy to treat several cardiomyopathies.

\section{Corneal epithelium and renal tubular cells}

MSCs were found to transfer functional mitochondria to human corneal epithelial cells, which improved mitochondrial bioenergetics in recipient cells, studied using extracellular flux analyzer. Comparison of ATP production, resting respiration and maximal respiration rates showed that rates of these bioenergetics parameters were found to be lower in rotenone-treated corneal epithelial cells (CECs) as compared to normal CECs. All these parameters were improved in CECs co-cultured with MSCs. It was demonstrated that mitochondria were transferred from MSCs to damaged CECs, which also protected them from rotenone-induced cell death and proliferation-inhibition [16]. This study suggests that mitochondrial transfer can have huge implications in treating alkali-eye burns and other eye related damages. It can further prove to be an efficient strategy to provide protection to cornea against oxidative stressinduced mitochondrial damage. Interestingly, this study has also suggested a two-way flow of cytoplasmic components between cells that trigger the response of stem cells to the differentiated cells. Intercellular communication between renal epithelial cells has also been shown through exchange of cytoplasmic materials [71]. Mitochondrial transfer from MSCs to renal tubular cells has been observed in a co-culture of rat renal tubular cells and human MSCs through intercellular connections [72].

\section{Brain cortical cells}

Intercellular communication and mitochondrial transfer from MSCs to rat cortical neurons has been observed in co-culture in in vitro system [73]. A prominent neuroprotective behavior of MSCs was established through mitochondrial transfer and overexpression of Miro1, followed by subsequent increase in brain derived neurotrophic factor. Furthermore, intravenous injection of MSCs post ischemia was found to mitigate the pathological symptoms of stroke and restoration of neurological activity [73]. The role of mitochondrial transfer in brain cells is also evident from a recent interesting study that has shown transfer of mitochondria from astrocytes to neurons as a means of neuroglia cellular cross-talk in alleviation of stroke [47]. There is a pressing need to further investigate the role of mitochondrial transfer from stem cell to brain that can aid in devising better therapeutic strategies.

WJ-MSCs were co-cultured with mitochondrial DNAdepleted cells. Importantly, these cells regained the expression of mtDNA-encoded proteins and showed functional oxygen consumption and respiratory control, as well as the activity of electron transport chain complexes I, II, III and IV. In addition, metabolic shifting and ETC complex Vinhibitor-sensitive ATP production were also recovered. Furthermore, cellular behaviors like attachment-free proliferation, aerobic viability and OXPHOS-dependent cellular motility were also recovered after mitochondrial transfer by WJMSCs [74]. This study, along with the others cited above, provide strong evidence that MSCs can serve as potential therapeutics to ameliorate diseases with mitochondrial dysfunction, through donation of healthy mitochondria.

\section{Antimicrobial activity of MSCs through mitochondrial transfer}

MSCs are known to aid in regeneration through multiple ways that include anti-oxidant property, anti-inflammatory activities of MSCs and differentiation to regenerate damaged tissue. MSCs also demonstrate ability to combat 
infection by transferring mitochondria to macrophages. This leads to modulation of phagocytic ability of macrophages through tunnel tube by enhancing their bioenergetics evidenced by increased ATP activity. This facilitates more efficient clearing of bacterial cells, thereby influencing innate immune response of these cells serving as an important mechanism in pre-clinical models of acute respiratory distress syndrome (ARDS) and sepsis [75]. To establish that anti-microbial effect of MSCs is mediated by alveolar macrophage, effect of MSCs was determined in normal $E$. coli infected model with animal model treated with intranasal Clodronate Lipososmes (CL) that completely abrogated alveolar macrophages (AM). It was found that administration of MSC treatment in AM depleted mice was not able to restore levels of several cytokines involved in antiinflammatory affects. But, in contrast MSCs could restore levels of cytokines in normal mice suggesting that the antimicrobial activity of MSCs is mediated through macrophages. Interestingly, macrophage phagocytosis of MSCs or fusion of MSC and macrophage was not observed in monocytes or neutrophils. This suggests that mitochondria transfer as the only means for enhanced engulfment by macrophages by both confocal and flow cytometry analysis. It was also observed that non-contact transfer of mitochondria through microvesicles or exosomes significantly enhanced mitochondria transfer and phagocytosis index of macrophages suggesting it as another critical mechanism for mitochondrial transfer. Another study by Phinney et al., has also shown that mitochondria transfer through microvesicles to macrophages and support paracrine and immune reaction. However, further investigation to better understand the role of mitochondria in anti-microbial and immune-modulation can provide better insights of multilevel reparative contributions of mitochondria in maintenance of cellular health post transfer to recipient cells.

\section{Inhibition of mitochondrial transfer: A potential target for anti-tumor therapies}

Mitochondria are the key regulators of cellular bioenergetics and metabolism that greatly impacts cancer progression [27]. Many researchers have pointed out mitochondria as a potential target for cancer therapies [7, 76]. In terms of MSCs, many studies have reported transfer of mitochondria through stem cells to tumor cells via tunnel tube formation contributing to their chemo resistance and proliferation $[18,43]$. A very interesting study by Dong et al., have demonstrated that metastatic melanoma cells without mitochondrial DNA and with defective respiratory function were unable to form tumours. However, upon acquiring intact mitochondria along with their mtDNA from their host through horizontal transfer, the cellular respiration is restored. This was shown to be critical in tumor generation in mice [77]. It has been found that mitochondrial transfer from bone marrow stem cells renders survival advantage to acute myeloid leukemic cells thereby making them resistant to chemotherapy [18]. In a recent study, it was shown that inhibition of ICAM-1 an adhesion molecule prevented mitochondria transfer from MSCs to Jurkat Cells treated with chemotherapeutic drug. This prevented tunnel tube formation and stopped transfer of mitochondria from MSCs to Jurkat cells and eventually induced chemotherapy induced cell-death [74]. Thus, inhibition of mitochondrial transfer can serve as a potential therapy target for cancer, such as selective blockage of tunnel tube formation for preventing mitochondrial transfer $[11,78]$. However, in view of the importance of aerobic glycolysis in cancer cells it remains possible that altering mitochondrial transfer to cancer cells may have context dependent effects and this merits further investigation [79].

\section{Challenges and future scope}

Mitochondrial dysfunction is usually linked to a variety of diseases and as discussed above, mitochondrial transfer from healthy MSCs can potentially serve as an effective remedy for alleviation of such diseases. Despite significant advancements in this field, mitochondrial transfer has yet not become a therapeutic strategy for treatment purposes. The optimization of MSC donor type, dose, and development of cell delivery techniques present unexplored challenges for medical intervention to treat pathological conditions with mitochondrial dysfunction. In addition, very few research groups have investigated if mitochondrial transfer is self-sufficient for repair or requires synergistic release of other paracrine effects such as immunomodulatory factors and exosomes. It is critical to analyze mitochondria transfer holistically by comparing roles of secretome, exosome and mitochondrial transfer acting together and in isolation to better understand the regenerative mechanisms. Ahmad et al., demonstrated that paracrine mediators such as NO, TGF- $\beta$, IL-10 and PGE2 were unaffected by Miro1 overexpression, suggesting that mitochondrial transfer can work independent of paracrine effects [34]. In another study by Zhang et al., it was suggested that both mitochondrial transfer and paracrine factors provide protective advantage to cardiomyocytes against stress conditions. But, both these processes work independent of each other [41]. MSCs were seen to manage intracellular oxidative stress by targeting depolarized mitochondria to the plasma membrane via microvesicles. These are then taken up by macrophages, resulting in enhanced bioenergetics in recipient cells. An orchestra of proteins are then activated by release of miRNA from exosomes that further de-sensitize uptake of mitochondria suggesting a cross-talk between mitochondria and other paracrine factors [75]. But, combinatorial effect of these mechanisms in other models is largely unexplored and a systematic study is important to completely understand these mechanisms and their synergy. 


\section{Conclusion}

Mitochondrial transfer through MSCs has shown promising therapeutic results in varied disease conditions through various modes of action as discussed in this review. In addition, it is widely known that intercellular organelle communication aids in maintenance of cellular homeostasis, particularly in terms of energy metabolism and cellular bioenergetics regulation [56, 71, 80]. Several environmental cues such as oxidative stress, release of DAMPs and mtDNA from injured cell trigger mitochondria transfer from MSCs. This suggests that modulation of the extracellular milieu can regulate mitochondrial transfer that is evident from enhanced mitochondrial transfer from BMMSCs upon treatment of anti-oxidants such as of $\mathrm{N}$-acetylL-cysteine (NAC) and L-ascorbic acid 2-phosphate (AAP) [81]. Such innovative strategies can further be employed for efficient treatments using mitochondrial transfer. Furthermore, inhibition of mitochondrial transfer to tumor cells can serve as a novel strategy for cancer treatment [7, 18]. The use of isolated and artificial mitochondria for treatment of diseases appears to be a lucrative future therapy [55]. Further investigations are imperative to use artificial mitochondria for treatment of diseases. The optimum dose, packaging, delivery methods and ethical issues for using isolated mitochondria have yet not been studied. It is interesting to note that isolated mitochondria may emerge as an off-the shelf therapy in future, as many researchers are now exploring the role of isolated mitochondria in regenerative medicine.

\section{Abbreviations \\ AAP: ascorbic acid 2-phosphate; AD-MSCs: adipose derived stem cell; AM: alveolar macrophages; ARDS: Acute Respiratory Distress Syndrome; BM- MSCs: Bone marrow mesenchymal stem cell; CL: Clodronate Lipososmes; DAMPS: damage associated molecular patterns; DP-MSCs: Dental Pulp derived mesenchymal stem cell; iPSCs: Induced Pluripotent Stem Cells; \\ MSCs: Mesenchymal stem cells; WJ-MSCs: Wharton's Jelly, mesenchymal stemcel}

\section{Acknowledgements}

We thank Sonali Rawat for contribution to diagrammatic representation of mitochondrial transfer in Fig. 2 and Fig. 3 of this review. We thank Manish Kumar for assisting with confocal microscope imaging. We also thank Dr. Swati Midha, Dr. Manu Dalela and Manisha Singh for their suggestions and proofreading this review.

\section{Funding}

Funds obtained from Department of Biotechnology, Ministry of Science and Technology, India.

\section{Availability of data and materials}

Data and materials related to this work are available upon request.

\section{Authors' contributions}

SP and SM have jointly contributed to writing the review. SP, RC, AA and SM have jointly revised the review in its current form. All authors read and approved the final manuscript.

\section{Ethics approval and consent to participate}

This study was ethically approved by the Institutional Committee for Stem Cell Research (IC-SCR), All India Institute of Medical Sciences (AlIMS), New Delhi.

\section{Consent for publication}

All authors read the final manuscript and approved manuscript for publication. Table 1 represents data from existing literature. Written informed consent was obtained from the patient for accompanying images (Figs. 1 and 4) in this review. The consent form is held by the authors/by the authors' institution and is available for review by the Editor-in-Chief.

\section{Competing interests}

The authors declare that they have no competing interests.

\section{Publisher's Note}

Springer Nature remains neutral with regard to jurisdictional claims in published maps and institutional affiliations.

Received: 1 November 2017 Accepted: 14 March 2018

Published online: 30 March 2018

\section{References}

1. Wei $X$, et al. Mesenchymal stem cells: a new trend for cell therapy. Acta Pharmacol Sin. 2013;34(6):747-54

2. Dimarino AM, Caplan Al, Bonfield TL. Mesenchymal stem cells in tissue repair. Front Immunol. 2013:4:201.

3. Patel DM, Shah J, Srivastava AS. Therapeutic potential of mesenchymal stem cells in regenerative medicine. Stem Cells Int. 2013:2013:496218.

4. Liang $X$, et al. Paracrine mechanisms of mesenchymal stem cell-based therapy: current status and perspectives. Cell Transplant. 2014;23(9):1045-59.

5. Liu CY, Lee CF, Wei YH. Role of reactive oxygen species-elicited apoptosis in the pathophysiology of mitochondrial and neurodegenerative diseases associated with mitochondrial DNA mutations. J Formos Med Assoc. 2009; 108(8):599-611.

6. Mabalirajan $U$, et al. Mitochondrial structural changes and dysfunction are associated with experimental allergic asthma. J Immunol. 2008;181(5):3540-8.

7. Loureiro R, et al. Mitochondria in cancer stem cells: a target for therapy. Recent Pat Endocr Metab Immune Drug Discov. 2013;7(2):102-14.

8. Spees JL, et al. Mitochondrial transfer between cells can rescue aerobic respiration. Proc Natl Acad Sci U S A. 2006;103(5):1283-8.

9. Rustom A, et al. Nanotubular highways for intercellular organelle transport. Science. 2004;303(5660):1007-10.

10. Onfelt B, et al. Cutting edge: membrane nanotubes connect immune cells. J Immunol. 2004;173(3):1511-3.

11. Bukoreshtliev NV, et al. Selective block of tunneling nanotube (TNT) formation inhibits intercellular organelle transfer between PC12 cells. FEBS Lett. 2009;583(9):1481-8.

12. Gerdes HH, Bukoreshtliev NV, Barroso JF. Tunneling nanotubes: a new route for the exchange of components between animal cells. FEBS Lett. 2007:581(11):2194-201.

13. Abounit S, Zurzolo C. Wiring through tunneling nanotubes-from electrical signals to organelle transfer. J Cell Sci. 2012:125(Pt 5):1089-98.

14. Cho YM, et al. Mesenchymal stem cells transfer mitochondria to the cells with virtually no mitochondrial function but not with pathogenic mtDNA mutations. PLoS One. 2012;7(3):e32778

15. Islam MN, et al. Mitochondrial transfer from bone-marrow-derived stromal cells to pulmonary alveoli protects against acute lung injury. Nat Med. 2012;18(5):759-65.

16. Jiang $D$, et al. Mitochondrial transfer of mesenchymal stem cells effectively protects corneal epithelial cells from mitochondrial damage. Cell Death Dis. 2016;7(11):e2467.

17. Figeac F, et al. Nanotubular crosstalk with distressed cardiomyocytes stimulates the paracrine repair function of mesenchymal stem cells. Stem Cells. 2014;32(1):216-30.

18. Moschoi $R$, et al. Protective mitochondrial transfer from bone marrow stromal cells to acute myeloid leukemic cells during chemotherapy. Blood. 2016;128(2):253-64.

19. Lin HY, et al. Mitochondrial transfer from Wharton's jelly-derived mesenchymal stem cells to mitochondria-defective cells recaptures impaired mitochondrial function. Mitochondrion. 2015;22:31-44.

20. Chen $C T$, et al. Coordinated changes of mitochondrial biogenesis and antioxidant enzymes during osteogenic differentiation of human mesenchymal stem cells. Stem Cells. 2008;26(4):960-8.

21. Wanet $\mathrm{A}$, et al. Mitochondrial remodeling in hepatic differentiation and dedifferentiation. Int J Biochem Cell Biol. 2014;54:174-85. 
22. Nagley $\mathrm{P}$, Linnane AW. Biogenesis of mitochondria. XXI. Studies on the nature of the mitochondrial genome in yeast: the degenerative effects of ethidium bromide on mitochondrial genetic information in a respiratory competent strain. J Mol Biol. 1972;66(1):181-93.

23. Youle RJ, Narendra DP. Mechanisms of mitophagy. Nat Rev Mol Cell Biol. 2011;12(1):9-14

24. Watkins J, Basu S, Bogenhagen DF. A quantitative proteomic analysis of mitochondrial participation in p19 cell neuronal differentiation. J Proteome Res. 2008;7(1):328-38.

25. Ding WX, Yin XM. Mitophagy: mechanisms, pathophysiological roles, and analysis. Biol Chem. 2012;393(7):547-64

26. Lonergan T, Bavister B, Brenner C. Mitochondria in stem cells. Mitochondrion. 2007:7(5):289-96.

27. Vega-Naredo I, et al. Mitochondrial metabolism directs stemness and differentiation in P19 embryonal carcinoma stem cells. Cell Death Differ. 2014;21(10):1560-74

28. Nishitai G, et al. Stress induces mitochondria-mediated apoptosis independent of SAPKJJNK activation in embryonic stem cells. J Biol Chem. 2004;279(3):1621-6.

29. Arranz L, Urbano-Ispizua A, Mendez-Ferrer S. Mitochondria underlie different metabolism of hematopoietic stem and progenitor cells. Haematologica. 2013;98(7):993-5.

30. $\mathrm{Xu} \mathrm{X,} \mathrm{et} \mathrm{al.} \mathrm{Mitochondrial} \mathrm{regulation} \mathrm{in} \mathrm{pluripotent} \mathrm{stem} \mathrm{cells.} \mathrm{Cell} \mathrm{Metab.}$ 2013;18(3):325-32

31. Armstrong $L$, et al. Human induced pluripotent stem cell lines show stress defense mechanisms and mitochondrial regulation similar to those of human embryonic stem cells. Stem Cells. 2010;28(4):661-73.

32. Bukowiecki R, Adjaye J, Prigione A. Mitochondrial function in pluripotent stem cells and cellular reprogramming. Gerontology. 2014;60(2):174-82.

33. Acquistapace $\mathrm{A}$, et al. Human mesenchymal stem cells reprogram adult cardiomyocytes toward a progenitor-like state through partial cell fusion and mitochondria transfer. Stem Cells. 2011;29(5):812-24.

34. Ahmad T, et al. Miro 1 knockdown in stem cells inhibits mitochondrial donation mediated rescue of bronchial epithelial injury. Biophys J. 2014;104(2):659a

35. Liu K, et al. Mesenchymal stem cells rescue injured endothelial cells in an in vitro ischemia-reperfusion model via tunneling nanotube like structuremediated mitochondrial transfer. Microvasc Res. 2014;92:10-8.

36. Galluzzi L, Kepp O, Kroemer G. Mitochondria: master regulators of danger signalling. Nat Rev Mol Cell Biol. 2012;13(12):780-8.

37. Nakahira K, Hisata S, Choi AM. The roles of mitochondrial damage-associated molecular patterns in diseases. Antioxid Redox Signal. 2015;23(17):1329-50.

38. Zhang Q, et al. Circulating mitochondrial DAMPs cause inflammatory responses to injury. Nature. 2010;464(7285):104-7.

39. Maeda A, Fadeel B. Mitochondria released by cells undergoing TNF-alphainduced necroptosis act as danger signals. Cell Death Dis. 2014;5:e1312.

40. Mahrouf-Yorgov $\mathrm{M}$, et al. Mesenchymal stem cells sense mitochondria released from damaged cells as danger signals to activate their rescue properties. Cell Death Differ. 2017;24(7):1224-38.

41. Zhang Y, et al. iPSC-MSCs with High Intrinsic MIRO1 and Sensitivity to TNFalpha Yield Efficacious Mitochondrial Transfer to Rescue AnthracyclineInduced Cardiomyopathy. Stem Cell Reports. 2016;7(4):749-63.

42. Torralba D, Baixauli F, Sanchez-Madrid F. Mitochondria know no boundaries: mechanisms and functions of intercellular mitochondrial transfer. Front Cell Dev Biol. 2016:4:107.

43. Pasquier J, et al. Preferential transfer of mitochondria from endothelial to cancer cells through tunneling nanotubes modulates chemoresistance. J Transl Med. 2013;11:94

44. Kaneyuki $Y$, Yoshino $H$, Kashiwakura I. Involvement of intracellular reactive oxygen species and mitochondria in the radiosensitivity of human hematopoietic stem cells. J Radiat Res. 2012;53(1):145-50.

45. Marlein $\mathrm{CR}$, et al. NADPH oxidase-2 derived superoxide drives mitochondria transfer from bone marrow stromal cells to leukemic blasts. Blood. 2017; 130(14):1649-60.

46. Huang PJ, et al. Transferring Xenogenic mitochondria provides neural protection against ischemic stress in ischemic rat brains. Cell Transplant. 2016;25(5):913-27

47. Hayakawa K, et al. Transfer of mitochondria from astrocytes to neurons after stroke. Nature. 2016;535(7613):551-5.

48. Wang $X$, Gerdes HH. Transfer of mitochondria via tunneling nanotubes rescues apoptotic PC12 cells. Cell Death Differ. 2015;22(7):1181-91.
49. Phinney DG, et al. Mesenchymal stem cells use extracellular vesicles to outsource mitophagy and shuttle microRNAs. Nat Commun. 2015:6:8472.

50. Oka T, et al. Mitochondrial DNA that escapes from autophagy causes inflammation and heart failure. Nature. 2012:485(7397):251-5.

51. Boudreau LH, et al. Platelets release mitochondria serving as substrate for bactericidal group IIA-secreted phospholipase A2 to promote inflammation. Blood. 2014;124(14):2173-83.

52. Weinberg SE, Sena LA, Chandel NS. Mitochondria in the regulation of innate and adaptive immunity. Immunity. 2015;42(3):406-17.

53. Koyanagi $M$, et al. Cell-to-cell connection of endothelial progenitor cells with cardiac myocytes by nanotubes: a novel mechanism for cell fate changes? Circ Res. 2005;96(10):1039-41.

54. Caicedo A, et al. MitoCeption as a new tool to assess the effects of mesenchymal stem/stromal cell mitochondria on cancer cell metabolism and function. Sci Rep. 2015:5:9073.

55. Caicedo A, et al. Artificial mitochondria transfer: current challenges, advances, and future applications. Stem Cells Int. 2017:2017:7610414.

56. Sinha $P$, et al. Intercellular mitochondrial transfer: bioenergetic crosstalk between cells. Curr Opin Genet Dev. 2016;38:97-101.

57. Sinclair KA, et al. Characterization of intercellular communication and mitochondrial donation by mesenchymal stromal cells derived from the human lung. Stem Cell Res Ther. 2016;7(1):91.

58. Pacak CA, et al. Actin-dependent mitochondrial internalization in cardiomyocytes: evidence for rescue of mitochondrial function. Biol Open. 2015;4(5):622-6.

59. Kitani T, et al. Internalization of isolated functional mitochondria: involvement of macropinocytosis. J Cell Mol Med. 2014;18(8):1694-703.

60. Li X, et al. Mitochondrial transfer of induced pluripotent stem cell-derived mesenchymal stem cells to airway epithelial cells attenuates cigarette smoke-induced damage. Am J Respir Cell Mol Biol. 2014;51(3):455-65.

61. Barbash IM, et al. Systemic delivery of bone marrow-derived mesenchymal stem cells to the infarcted myocardium: feasibility, cell migration, and body distribution. Circulation. 2003:108(7):863-8.

62. Vallabhaneni KC, Haller H, Dumler I. Vascular smooth muscle cells initiate proliferation of mesenchymal stem cells by mitochondrial transfer via tunneling nanotubes. Stem Cells Dev. 2012;21(17):3104-13.

63. Plotnikov EY, et al. Cell-to-cell cross-talk between mesenchymal stem cells and cardiomyocytes in co-culture. J Cell Mol Med. 2008;12(5A):1622-31.

64. Ikeda $Y$, et al. Molecular mechanisms mediating mitochondrial dynamics and mitophagy and their functional roles in the cardiovascular system. J Mol Cell Cardiol. 2015;78:116-22.

65. Masuzawa A, et al. Transplantation of autologously derived mitochondria protects the heart from ischemia-reperfusion injury. Am J Physiol Heart Circ Physiol. 2013;304(7):H966-82.

66. Nair $\mathrm{V}$, et al. Efficacy of stem cell in improvement of left ventricular function in acute myocardial infarction-MI3 trial. Indian J Med Res. 2015;142(2):165-74.

67. Cselenyak A, et al. Mesenchymal stem cells rescue cardiomyoblasts from cell death in an in vitro ischemia model via direct cell-to-cell connections. BMC Cell Biol. 2010;11:29.

68. McCully JD, et al. Injection of isolated mitochondria during early reperfusion for cardioprotection. Am J Physiol Heart Circ Physiol. 2009:296(1):H94-H105.

69. Han $\mathrm{H}$, et al. Bone marrow-derived mesenchymal stem cells rescue injured H9c2 cells via transferring intact mitochondria through tunneling nanotubes in an in vitro simulated ischemia/reperfusion model. Mol Med Rep. 2016; 13(2):1517-24

70. Ma Z, et al. Mesenchymal stem cell-cardiomyocyte interactions under defined contact modes on laser-patterned biochips. PLoS One. 2013;8(2):e56554.

71. Domhan S, et al. Intercellular communication by exchange of cytoplasmic material via tunneling nano-tube like structures in primary human renal epithelial cells. PLoS One. 2011;6(6):e21283.

72. Plotnikov EY, et al. Cytoplasm and organelle transfer between mesenchymal multipotent stromal cells and renal tubular cells in co-culture. Exp Cell Res. 2010;316(15):2447-55

73. Babenko VA, et al. Improving the post-stroke therapeutic potency of mesenchymal multipotent stromal cells by Cocultivation with cortical neurons: the role of crosstalk between cells. Stem Cells Transl Med. 2015:4(9):1011-20.

74. Yang $X$, et al. Mesenchymal stem cells derived from Wharton jelly of the human umbilical cord ameliorate damage to human endometrial stromal cells. Fertil Steril. 2011;96(4):1029-36.

75. Jackson MV et al. Mitochondrial transfer via tunneling nanotubes is an important mechanism by which mesenchymal stem cells enhance 
macrophage phagocytosis in the in vitro and in vivo models of ARDS. Stem Cells. 2016;34(8):2210-23.

76. Kubohara Y, et al. Mitochondria are the target organelle of differentiationinducing factor-3, an anti-tumor agent isolated from Dictyostelium discoideum [corrected]. PLoS One. 2013;8(8):e72118.

77. Dong $L F$, et al. Horizontal transfer of whole mitochondria restores tumorigenic potential in mitochondrial DNA-deficient cancer cells. elife. 2017;6

78. Takahashi A, et al. Ectopically expressed variant form of sperm mitochondriaassociated cysteine-rich protein augments tumorigenicity of the stem cell population of lung adenocarcinoma cells. PLoS One. 2013;8(11):e69095.

79. Liberti MV, Locasale JW. The Warburg effect: how does it benefit Cancer cells? Trends Biochem Sci. 2016;41(3):211-8.

80. Vignais ML, et al. Cell connections by tunneling nanotubes: effects of mitochondrial trafficking on target cell metabolism, homeostasis, and response to therapy. Stem Cells Int. 2017:2017:6917941.

81. Li CJ, et al. Enhancement of mitochondrial transfer by antioxidants in human mesenchymal stem cells. Oxidative Med Cell Longev. 2017;2017:8510805.

Submit your next manuscript to BioMed Central and we will help you at every step:

- We accept pre-submission inquiries

- Our selector tool helps you to find the most relevant journal

- We provide round the clock customer support

- Convenient online submission

- Thorough peer review

- Inclusion in PubMed and all major indexing services

- Maximum visibility for your research

Submit your manuscript at www.biomedcentral.com/submit
Biomed Central 